\title{
Carga de trabalho de enfermagem requerida por adultos, idosos e muito idosos em Unidade de Terapia Intensiva
}

\author{
NURSING WORKLOAD AMONG ADULTS, ELDERLY AND VERY ELDERLY PATIENTS \\ IN THE INTENSIVE CARE UNIT
}

CARGA DE TRABAJO DE ENFERMERÍA RELACIONADAAADULTOS, ANCIANOSY
MUY ANCIANOS EN TERAPIA INTENSIVA

Regina Marcia Cardoso de Sousa ${ }^{1}$, Katia Grillo Padilha², Lilia de Souza Nogueira ${ }^{3}$, Ana Maria Kazue Miyadahira $^{4}$, Verônica Cunha Rodrigues de Oliveira ${ }^{5}$

\section{RESUMO}

O objetivo deste estudo foi comparar a carga de trabalho de enfermagem requerida pelos pacientes adultos, idosos e muito idosos, incluindo nas análises as intervenções realizadas e a evolução da carga de trabaIho entre admissão e alta. Estudo prospectivo, longitudinal, realizado em UTIs gerais de quatro hospitais do Município de São Paulo. Os resultados apontaram que, independente da idade, houve similaridade da carga de trabalho de enfermagem na admissão, bem como na evolução das demandas de cuidados dos pacientes. Diferenças entre os grupos foram observadas no NAS na alta da UTI e nos seguintes itens desse instrumento: Monitorização e controles e Procedimentos de higiene, na admissão, Suporte respiratório e Hiperalimentação intravenosa na alta e Mobilização e posicionamento tanto na alta como na admissão. Concluiuse, portanto, que a idade interferiu somente em aspectos específicos da carga de trabalho de enfermagem requeridas por pacientes internados em UTIs.

\section{DESCRITORES}

Carga de trabalho.

Enfermagem.

Unidades de Terapia Intensiva.

Grupos etários.

\begin{abstract}
The study's objectives were to compare nursing workload among adults, elderly and very elderly patients, including in the analyses the interventions and evolution of the workload between admission and discharge. This prospective longitudinal study involved 600 adult patients in general ICU in four city hospitals in Sao Paulo. The results showed that, independent of the age, it had the similarity of the nursing workload in the admission, as well as in the evolution of the patients' care. Differences between the groups were observed in the NAS on ICU's discharge and the following instrument's items: Monitoring and titration and Hygiene procedures in admission, Respiratory support and Intravenous hyperalimentation in discharge and Mobilization and positioning in discharge and admission. In conclusion, the results evidenced that the age interfered only in specific aspects of the nursing workload required by patients in ICUs.
\end{abstract}

\section{KEY WORDS}

Workload.

Nursing.

Intensive Care Units.

Age groups.

\section{RESUMEN}

El objetivo fue comparar la carga de trabajo de enfermería entre los pacientes adultos, ancianos y muy ancianos, incluyendo en las análisis las intervenciones y la evolución de la carga de trabajo entre admisión y alta. Estudio prospectivo longitudinal de 600 pacientes adultos internados en la $\mathrm{UCl}$ general de cuatro hospitales de São Paulo. Como resultados, independientemente de la edad, hubo similitud de la carga de trabajo de enfermería en la admisión y evolución de las demandas de cuidados. Diferencias entre los grupos fueron observadas en el NAS en la alta de la UCl y en los siguientes apartados de esta herramienta: Monitorización y controles y Procedimientos de higiene, en la admisión; Soporte ventilatorio e Iperalimentación intravenosa en la alta; y Movilización y posicionamiento en la alta y admisión. Se concluye, por tanto, que la edad interfiere sólo en aspectos especificos de la carga de trabajo en enfermería requeridos por los pacientes internados en la $\mathrm{UCl}$.

\author{
DESCRIPTORES \\ Carga de trabajo. \\ Enfermería. \\ Unidades de Terapia Intensiva. \\ Grupos por edad.
}

\footnotetext{
${ }^{1}$ Professora Associada Departamento de Enfermagem Médico Cirúrgica da Escola de Enfermagem da Universidade de São Paulo. São Paulo, SP, Brasil. vian@usp.br ${ }^{2}$ Professora Titular do Departamento de Enfermagem Médico Cirúrgica da Escola de Enfermagem da Universidade de São Paulo. São Paulo, SP, Brasil. kgpadilh@usp.br ${ }^{3}$ Enfermeira. Mestre e doutoranda pela Escola de Enfermagem da Universidade de São Paulo. São Paulo, SP, Brasil. lilianogueira@usp.br ${ }^{4}$ Professora Titular do Departamento de Enfermagem Médico Cirúrgica da Escola de Enfermagem da Universidade de São Paulo. São Paulo, SP, Brasil. seizom@uol.com.br ${ }^{5}$ Enfermeira. Mestranda pela Escola de Enfermagem da Universidade de São Paulo. São Paulo, SP, Brasil. vcr@usp.br 


\section{INTRODUÇÃO}

As Unidades de Terapia Intensiva (UTIs) são unidades de alto custo, visto que requerem a utilização de equipamentos de alta tecnologia, espaço físico diferenciado e pessoal altamente qualificado. Estudos apontam que o custo da mão de obra especializada de enfermagem é uma das principais fontes de consumo de recursos nesses ambientes, daí a necessidade de um adequado quantitativo de pessoal ${ }^{(1-4)}$. Nesse contexto, um dos desafios dos gestores dessas unidades é adequar o staff de enfermagem às demandas de cuidados exigidas pelos pacientes graves, que inclui contingente cada vez maior de idosos e muito idosos nas UTIs, em decorrência do envelhecimento da população mundial.

Segundo a Organização Mundial de Saúde (OMS) ${ }^{(5)}$, no período de 2005 a 2040, estima-se em 164\% o aumento da população de idosos com mais de 65 anos, em 301\% com mais de 85 e em $746 \%$ com idade maior do que 100 anos.

No Brasil, o número de idosos passou de 3 milhões, em 1960, para 7 milhões, em 1975, e 17 milhões, em 2006, um aumento de $600 \%$ em menos de cinqüenta anos. Ademais, projeções conservadoras indicam que, em 2020, o Brasil será o sexto país do mundo em número de idosos, com um contingente superior a 30 milhões de pessoas ${ }^{(6)}$.

Diante desse cenário e tendo em conta que o envelhecimento se caracteriza pela deteriorização gradual das reservas funcionais, aumento das comorbidades, incapacidades e diminuição da independência e autonomia, prevê-se número cada vez maior de pacientes com idades avançadas necessitando de atendimento à saúde em UT( ${ }^{(7-8)}$.

Embora existam controvérsias relacionadas à admissão de idosos em unidades críticas $^{(7-8)}$, uma vez na unidade, os pacientes exigem cuidados de enfermagem que deverão ser providos por um quantitativo de pessoal adequado. Nesse sentido, é de interesse conhecer como é a participação dos idosos nesse contexto e se pacientes adultos, de faixas etárias progressivas, consomem maior carga de trabalho de enfermagem para seus cuidados.

\section{OBJETIVO}

Frente a essas questões, este estudo tem por objetivo comparar a carga de trabalho de enfermagem requerida por pacientes adultos, idosos e muito idosos, incluindo nas análises as intervenções realizadas e a evolução da carga de trabalho nos momentos de admissão e alta das UTIs.

\section{MÉTODO}

Trata-se de um estudo prospectivo, longitudinal, de pacientes adultos internados em UTIs gerais de dois hospitais públicos e dois privados. Os hospitais foram selecionados considerando os seguintes critérios: localização no Município de São Paulo; porte médio, grande ou extra grande; presença de UTI geral e unidade intermediária. Além de clientela exclusivamente pediátrica, foram critérios de exclusão número de leitos de UTI inferior a $6 \%$ do número total de leitos hospitalares e menos de cinco leitos em unidade intermediária ${ }^{(9)}$.

A casuística compôs-se de 600 pacientes com idade $\geq$ 18 anos, admitidos nessas UTIs de agosto de 2006 a janeiro de 2007 e que permaneceram na unidade 24 horas ou mais.

Após a aprovação dos Comitês de Ética e Pesquisa (Pareceres n SMS52/2006; HU650/06; HSL2006/03 e AE06/ 510) e contato prévio com os enfermeiros responsáveis pelas UTIs, foi iniciada a coleta de dados nas unidades selecionadas. Diariamente, foi realizado busca de novos pacientes e acompanhamento dos que já se encontravam inseridos na pesquisa. Os pacientes tiveram seguimento até a saída da UTI, a fim de se obter dados das primeiras e últimas 24 horas de permanência na unidade.

A carga de trabalho de enfermagem foi mensurada pelo Nursing Activities Score $\mathrm{NAS}^{(2)}$, e o risco de morte foi estabelecido segundo o Simplified Acute Physiology Score II-SAPS $\|^{(10)}$ e Logistic Organ Dysfunction Score $-\operatorname{LODS}^{(11)}$.

O tratamento dos dados foi feito nos programas Stata for Windows 8.0 e SPSS 13.0 for Windows. Para comparar os grupos de pacientes segundo idade foram considerados três faixas etárias que incluíram indivíduos com idade: $\geq 18$ e $<60$ anos (adultos), $\geq 60$ e $<80$ anos (idosos) e $\geq$ 80 anos (muito idosos).

$\mathrm{Na}$ análise das intervenções descritas no NAS, os itens 2, 3, 5 e de 9 a 23 foram considerados variáveis dicotômicas e a distribuição dos pacientes foi realizada conforme a presença ou ausência das intervenções. Nos itens 1, 4, 6, 7 e 8, que apresentam sub-itens mutuamente excludentes, os pacientes foram distribuídos de acordo com a seguinte classificação: sub-item 1a ou 1b e 1c; $4 a$ ou $4 b$ e $4 c ; 6 a$ ou $6 b$ e $6 c ; 7$ a ou $7 b$ e 8 a ou 8 b e $8 c$.

Para comparar os grupos de indivíduos adultos, idosos e muito idosos, segundo as intervenções, foi utilizado o teste de associação Qui-Quadrado de Person. Quando o resultado desse teste indicou diferença estatisticamente significativa entre os grupos $(p \leq 0,05)$, foi realizada aná- 
lise complementar entre os pares de grupos: adultos versus idosos, idosos versus muito idosos e adultos versus muito idosos, a fim de identificar entre os três grupos os que diferiram.

A evolução da carga de trabalho dos pacientes durante o período de internação nas UTIs foi analisada considerando a diferença do escore total do NAS entre o primeiro e o último dia de internação do paciente na UTI (NAS admissão - NAS alta ou óbito).

Nessa análise, além dos valores médios do NAS (admissão e alta da UTI), os grupos foram comparados utilizando o teste Anova-OneWay. No caso de indicação de diferenças, foi aplicado o teste de Tukey para verificar entre quais grupos ela ocorreu. Todas as análises foram realizadas considerando-se $\alpha \leq 0,05$.

\section{RESULTADOS}

Nos 600 pacientes analisados houve predominância de pacientes do sexo masculino, representando 56,70\% do total dos internados nas UTIs. Observou-se uma variação de idade 18 a 97 anos, sendo que 46,50\% dos pacientes pertenciam à categoria entre $\geq 18$ e $<60$ anos ( 279 indivíduos), $36,00 \%$ entre $\geq 60$ e $<80$ anos (216) e $17,50 \% \geq 80$ anos (105). A idade média dos pacientes que compuseram a amostra foi de 60,76 e a mediana de 62 anos.

Dentre as características clínicas, a maior parte dos pacientes era proveniente do Pronto-Socorro ou ProntoAtendimento (36,34\%) ou Centro Cirúrgico (35,50\%). Na análise das comorbidades, observou-se que a categoria mais freqüente de antecedentes foi à relacionada às doenças do aparelho circulatório (58,00\%). A média de tempo de permanência nas UTIs foi de $8,90( \pm 10,90)$ dias e a mortalidade de 20,00\%. Daqueles que sobreviveram $(n=480), 64,60 \%$ tiveram alta para as unidades intermediárias e $32,90 \%$ foram transferidos diretamente às unidades de internação.

Quanto ao risco de morte na admissão nas UTIs, a média foi de $25,50 \%$ segundo o SAPS II e de $21,43 \%$ pelo LODS, valores próximos aos verificados no momento da saída dessas unidades, $23,14 \%$ e $20,73 \%$, respectivamente.

Tabela 1 - Medidas de tendência central na admissão e na alta dos pacientes internados nas UTIs (n=600) de acordo com a faixa etária São Paulo - 2006/2007

\begin{tabular}{|c|c|c|c|c|c|c|c|}
\hline \multirow{3}{*}{ Variáveis } & \multicolumn{6}{|c|}{ Faixas etárias } & \multirow{3}{*}{$\mathbf{p}^{*}$} \\
\hline & \multicolumn{2}{|c|}{$\begin{array}{c}\text { Adultos } \\
(\geq 18 \mathrm{e}<60)\end{array}$} & \multicolumn{2}{|c|}{$\begin{array}{c}\text { Idosos } \\
(\geq 60 \mathrm{e}<80)\end{array}$} & \multicolumn{2}{|c|}{$\begin{array}{l}\text { Muito idosos } \\
(\geq 80)\end{array}$} & \\
\hline & Média & DP & Média & DP & Média & DP & \\
\hline NAS admissão & 59,98 & 22,40 & 64,41 & 20,66 & 62,45 & 20,80 & 0,07 \\
\hline NAS alta & 50,37 & 14,56 & 55,85 & 16,77 & 53,39 & 16,17 & 0,00 \\
\hline
\end{tabular}

* Teste de Anova-OneWay

Conforme se observa na Tabela 1, o valor médio do NAS variou entre os grupos de 64,41 a 59,98, na admissão, e de 55,85 a 50,37 , na alta, sendo que o grupo de idosos manteve nos dois momentos o maior valor médio. Houve diferença estatisticamente significativa entre os grupos, em relação aos valores do NAS somente na alta do paciente das UTIs $(p=0,00)$. O teste de Tukey identificou que esta diferença ocorreu entre os grupos de adultos e idosos $(p=0,00)$.

Na Tabela 2 observa-se que os pacientes adultos, idosos e muito idosos diferiram na admissão em relação às seguintes intervenções: Monitorização e controles, Procedimentos de higiene e Mobilização e posicionamento.

Quanto à Monitorização e controles, na comparação dos pares de grupos pelo Qui-Quadrado, observou-se que essa diferença ocorreu entre adultos e idosos com valor de $p=0,01$. No entanto, o grupo de muito idosos não diferiu dos demais.

Os adultos apresentaram maior freqüência (55,56\%) do que os idosos $(43,81 \%)$ em relação ao item a (sinais vitais horários, cálculo e registro regular do balanço hídrico). $\mathrm{O}$ oposto ocorreu no item b e c (necessidade de presença à beira do leito para monitorização e controle por 2 horas ou mais em algum plantão, ou por 4 horas ou mais, no caso do item c) em que os idosos foram mais freqüentes (56,19\%), caracterizando que este grupo despendeu maior tempo da assistência de enfermagem relacionada à Monitorização e controles.

Quanto aos Procedimentos de higiene, a comparação dos pares identificou que o grupo de muito idosos diferiu tanto do grupo de adultos $(p=0,01)$, quanto dos idosos $(p=0,02)$.

Os muito idosos apresentaram maior freqüência (31,37\%) de procedimentos de higiene que duraram mais do que 2 horas (item b) ou mais do que 4 horas em algum plantão (item c), em relação aos outros grupos (18,89\% e 19,52\%), evidenciando que esse grupo necessitou de maior tempo de enfermagem relacionado a este tipo de assistência.

Quanto à Mobilização e posicionamento, os adultos diferiram dos idosos $(p=0,00)$ e dos muito idosos $(p=0,03)$ na comparação dos pares de grupos. A realização do procedimento mais do que 3 vezes em 24 horas ou com 2 enfermeiros em qualquer freqüência (item b) e com 3 ou mais enfermeiros (item c) foi mais freqüente nos grupos de idosos $(64,76 \%)$ e muito idosos $(55,88 \%)$ perante ao grupo de adultos $(42,96 \%)$. 
Tabela 2 - Distribuição dos pacientes internados nas UTIs $(n=582 *)$ de acordo com a faixa etária e tipo de intervenção realizada na admissão, conforme o NAS - São Paulo - 2006/2007

\begin{tabular}{|c|c|c|c|c|c|c|c|c|}
\hline \multirow{3}{*}{ Tipo de intervenções - NAS } & \multicolumn{7}{|c|}{ Faixas etárias } & \multirow{3}{*}{$\mathbf{p}^{* *}$} \\
\hline & \multirow[t]{2}{*}{ Categorias } & \multicolumn{2}{|c|}{$\begin{array}{c}\text { Adultos } \\
(\geq 18 \mathrm{e}<60)\end{array}$} & \multicolumn{2}{|c|}{$\begin{array}{c}\text { Idosos } \\
(\geq 60 \text { e }<80)\end{array}$} & \multicolumn{2}{|c|}{$\begin{array}{c}\text { Muito idosos } \\
(\geq \mathbf{8 0})\end{array}$} & \\
\hline & & $\mathbf{N}$ & $\%$ & $\mathbf{N}$ & $\%$ & $\mathbf{N}$ & $\%$ & \\
\hline 1- Monitorização e controles & $\begin{array}{c}1 \mathrm{a} \\
1 \mathrm{~b} \text { e c }\end{array}$ & $\begin{array}{l}150 \\
120\end{array}$ & $\begin{array}{l}55,56 \\
44,44\end{array}$ & $\begin{array}{c}92 \\
118\end{array}$ & $\begin{array}{l}43,81 \\
56,19\end{array}$ & $\begin{array}{l}52 \\
50\end{array}$ & $\begin{array}{l}50,98 \\
49,02\end{array}$ & 0,04 \\
\hline 2- Investigações laboratoriais & $\begin{array}{l}\text { Sim } \\
\text { Não }\end{array}$ & $\begin{array}{c}269 \\
1\end{array}$ & $\begin{array}{c}99,63 \\
0,37\end{array}$ & $\begin{array}{c}210 \\
-\end{array}$ & $\begin{array}{c}100,00 \\
-\end{array}$ & $\begin{array}{c}102 \\
-\end{array}$ & $\begin{array}{c}100,00 \\
-\end{array}$ & 0,56 \\
\hline 3-Medicação & $\begin{array}{l}\text { Sim } \\
\text { Não }\end{array}$ & $\begin{array}{c}269 \\
1\end{array}$ & $\begin{array}{c}99,63 \\
0,37\end{array}$ & $\begin{array}{c}209 \\
1\end{array}$ & $\begin{array}{c}99,52 \\
0,48\end{array}$ & $\begin{array}{c}102 \\
-\end{array}$ & $\begin{array}{c}100,0 \\
-\end{array}$ & 0,79 \\
\hline 4- Procedimentos de higiene & $\begin{array}{c}4 \mathrm{a} \\
4 \mathrm{~b} \text { e c }\end{array}$ & $\begin{array}{c}219 \\
51\end{array}$ & $\begin{array}{l}81,11 \\
18,89\end{array}$ & $\begin{array}{c}169 \\
41\end{array}$ & $\begin{array}{l}80,48 \\
19,52\end{array}$ & $\begin{array}{l}70 \\
32\end{array}$ & $\begin{array}{l}68,63 \\
31,37\end{array}$ & 0,02 \\
\hline 5- Cuidados com drenos & $\begin{array}{l}\text { Sim } \\
\text { Não }\end{array}$ & $\begin{array}{c}173 \\
97\end{array}$ & $\begin{array}{l}64,07 \\
35,93\end{array}$ & $\begin{array}{c}136 \\
74\end{array}$ & $\begin{array}{l}64,76 \\
35,24\end{array}$ & $\begin{array}{l}64 \\
38\end{array}$ & $\begin{array}{l}62,75 \\
37,25\end{array}$ & 0,94 \\
\hline 6- Mobilização e posicionamento & $\begin{array}{c}6 \mathrm{a} \\
6 \mathrm{~b} \text { e c }\end{array}$ & $\begin{array}{l}154 \\
116\end{array}$ & $\begin{array}{l}57,04 \\
42,96\end{array}$ & $\begin{array}{c}74 \\
136\end{array}$ & $\begin{array}{l}35,24 \\
64,76\end{array}$ & $\begin{array}{l}45 \\
57\end{array}$ & $\begin{array}{l}44,12 \\
55,88\end{array}$ & $\mathbf{0 , 0 0}$ \\
\hline $\begin{array}{l}\text { 7- Suporte e cuidado aos familiares e } \\
\text { pacientes }\end{array}$ & $\begin{array}{l}7 \mathrm{a} \\
7 \mathrm{~b}\end{array}$ & $\begin{array}{c}252 \\
18\end{array}$ & $\begin{array}{c}93,33 \\
6,67\end{array}$ & $\begin{array}{c}194 \\
16\end{array}$ & $\begin{array}{c}92,38 \\
7,62\end{array}$ & $\begin{array}{c}98 \\
4\end{array}$ & $\begin{array}{c}96,08 \\
3,92\end{array}$ & 0,46 \\
\hline 8- Tarefas administrativas e gerenciais & $\begin{array}{c}8 \mathrm{a} \\
8 \mathrm{~b} \text { e c }\end{array}$ & $\begin{array}{c}194 \\
76\end{array}$ & $\begin{array}{l}71,85 \\
28,15\end{array}$ & $\begin{array}{c}140 \\
70\end{array}$ & $\begin{array}{l}66,67 \\
33,33\end{array}$ & $\begin{array}{l}74 \\
28\end{array}$ & $\begin{array}{l}72.55 \\
27,45\end{array}$ & 0,42 \\
\hline 9- Suporte respiratório & $\begin{array}{l}\text { Sim } \\
\text { Não }\end{array}$ & $\begin{array}{c}193 \\
77\end{array}$ & $\begin{array}{l}71,48 \\
28,52\end{array}$ & $\begin{array}{c}169 \\
41\end{array}$ & $\begin{array}{l}80,48 \\
19,52\end{array}$ & $\begin{array}{l}74 \\
28\end{array}$ & $\begin{array}{l}72,55 \\
27,45\end{array}$ & 0,06 \\
\hline 10- Cuidado com vias aéreas artificiais & $\begin{array}{l}\text { Sim } \\
\text { Não }\end{array}$ & $\begin{array}{c}94 \\
176\end{array}$ & $\begin{array}{l}34,81 \\
65,19\end{array}$ & $\begin{array}{c}76 \\
134\end{array}$ & $\begin{array}{l}36,19 \\
63,81\end{array}$ & $\begin{array}{l}44 \\
58\end{array}$ & $\begin{array}{l}43,14 \\
56,86\end{array}$ & 0,32 \\
\hline $\begin{array}{l}\text { 11- Tratamento para melhora da } \\
\text { função pulmonar }\end{array}$ & $\begin{array}{l}\text { Sim } \\
\text { Não }\end{array}$ & $\begin{array}{c}97 \\
173\end{array}$ & $\begin{array}{l}35,93 \\
64,07\end{array}$ & $\begin{array}{c}69 \\
141\end{array}$ & $\begin{array}{l}32,86 \\
67,14\end{array}$ & $\begin{array}{l}39 \\
63\end{array}$ & $\begin{array}{l}38,24 \\
61,76\end{array}$ & 0,61 \\
\hline 12- Medicação vasoativa & $\begin{array}{l}\text { Sim } \\
\text { Não }\end{array}$ & $\begin{array}{c}76 \\
194\end{array}$ & $\begin{array}{l}28,15 \\
71,85\end{array}$ & $\begin{array}{c}72 \\
138\end{array}$ & $\begin{array}{l}34,29 \\
65,71\end{array}$ & $\begin{array}{l}28 \\
74\end{array}$ & $\begin{array}{l}27,45 \\
72,55\end{array}$ & 0,28 \\
\hline $\begin{array}{l}\text { 13- Reposição intravenosa de grandes } \\
\text { perdas de fluidos }\end{array}$ & $\begin{array}{l}\text { Sim } \\
\text { Não }\end{array}$ & $\begin{array}{c}27 \\
243\end{array}$ & $\begin{array}{l}10,00 \\
90,00\end{array}$ & $\begin{array}{c}19 \\
191\end{array}$ & $\begin{array}{c}9,05 \\
90,95\end{array}$ & $\begin{array}{c}6 \\
96\end{array}$ & $\begin{array}{c}5,88 \\
94,12\end{array}$ & 0,46 \\
\hline 14- Monitorização de átrio esquerdo & $\begin{array}{l}\text { Sim } \\
\text { Não }\end{array}$ & $\begin{array}{c}3 \\
267\end{array}$ & $\begin{array}{l}1,11 \\
98,89\end{array}$ & $\begin{array}{c}5 \\
205\end{array}$ & $\begin{array}{c}2,38 \\
97,62\end{array}$ & $\begin{array}{c}1 \\
101\end{array}$ & $\begin{array}{c}0,98 \\
99,02\end{array}$ & 0,47 \\
\hline 15- Reanimação cardiorrespiratória & $\begin{array}{l}\text { Sim } \\
\text { Não }\end{array}$ & $\begin{array}{c}4 \\
266\end{array}$ & $\begin{array}{c}1,48 \\
98,52\end{array}$ & $\begin{array}{c}3 \\
207\end{array}$ & $\begin{array}{c}1,43 \\
98,57\end{array}$ & $\begin{array}{c}- \\
102\end{array}$ & $\begin{array}{c}- \\
100,00\end{array}$ & 0,47 \\
\hline 16- Técnicas de hemofiltração & $\begin{array}{l}\text { Sim } \\
\text { Não }\end{array}$ & $\begin{array}{c}20 \\
250\end{array}$ & $\begin{array}{c}7,41 \\
92,59\end{array}$ & $\begin{array}{c}12 \\
198\end{array}$ & $\begin{array}{c}5,71 \\
94,29\end{array}$ & $\begin{array}{c}6 \\
96\end{array}$ & $\begin{array}{c}5,88 \\
94,12\end{array}$ & 0,73 \\
\hline 17- Medida quantitativa do débito urinário & $\begin{array}{l}\text { Sim } \\
\text { Não }\end{array}$ & $\begin{array}{c}254 \\
16\end{array}$ & $\begin{array}{c}94,07 \\
5,93\end{array}$ & $\begin{array}{c}197 \\
13\end{array}$ & $\begin{array}{c}93,81 \\
6,19\end{array}$ & $\begin{array}{c}94 \\
8\end{array}$ & $\begin{array}{c}92,16 \\
7,84\end{array}$ & 0,79 \\
\hline 18- Medida de pressão intracraniana & $\begin{array}{l}\text { Sim } \\
\text { Não }\end{array}$ & $\begin{array}{c}10 \\
260\end{array}$ & $\begin{array}{c}3,70 \\
96,30\end{array}$ & $\begin{array}{c}2 \\
208\end{array}$ & $\begin{array}{c}0,95 \\
99,05\end{array}$ & $\begin{array}{c}1 \\
101\end{array}$ & $\begin{array}{c}0,98 \\
99,02\end{array}$ & 0,08 \\
\hline $\begin{array}{l}\text { 19- Tratamento de acidose/alcalose } \\
\text { metabólica complicada }\end{array}$ & $\begin{array}{l}\text { Sim } \\
\text { Não }\end{array}$ & $\begin{array}{c}4 \\
266\end{array}$ & $\begin{array}{c}1,48 \\
98,52\end{array}$ & $\begin{array}{c}5 \\
205\end{array}$ & $\begin{array}{c}2,38 \\
97,62\end{array}$ & $\begin{array}{c}4 \\
98\end{array}$ & $\begin{array}{c}3,92 \\
96,08\end{array}$ & 0,36 \\
\hline 20- Hiperalimentação intravenosa & $\begin{array}{l}\text { Sim } \\
\text { Não }\end{array}$ & $\begin{array}{c}13 \\
257\end{array}$ & $\begin{array}{c}4,81 \\
95,19\end{array}$ & $\begin{array}{c}16 \\
194\end{array}$ & $\begin{array}{c}7,62 \\
92,38\end{array}$ & $\begin{array}{c}7 \\
95\end{array}$ & $\begin{array}{c}6,86 \\
93,14\end{array}$ & 0,43 \\
\hline 21- Alimentação enteral & $\begin{array}{l}\text { Sim } \\
\text { Não }\end{array}$ & $\begin{array}{c}58 \\
212\end{array}$ & $\begin{array}{l}21,48 \\
78,52\end{array}$ & $\begin{array}{c}52 \\
158\end{array}$ & $\begin{array}{l}24,76 \\
75,24\end{array}$ & $\begin{array}{l}32 \\
70\end{array}$ & $\begin{array}{l}31,37 \\
68,63\end{array}$ & 0,14 \\
\hline 22- Intervenções específicas na unidade & $\begin{array}{l}\text { Sim } \\
\text { Não }\end{array}$ & $\begin{array}{c}18 \\
252\end{array}$ & $\begin{array}{c}6,67 \\
93,33\end{array}$ & $\begin{array}{c}22 \\
188\end{array}$ & $\begin{array}{l}10,48 \\
89,52\end{array}$ & $\begin{array}{c}8 \\
94\end{array}$ & $\begin{array}{c}7,84 \\
92,16\end{array}$ & 0,32 \\
\hline 23- Intervenções específicas fora da unidade & $\begin{array}{l}\text { Sim } \\
\text { Não }\end{array}$ & $\begin{array}{c}16 \\
254\end{array}$ & $\begin{array}{c}5,93 \\
94,07\end{array}$ & $\begin{array}{c}10 \\
200\end{array}$ & $\begin{array}{c}4,76 \\
95,24\end{array}$ & $\begin{array}{c}1 \\
101\end{array}$ & $\begin{array}{c}0,98 \\
99,02\end{array}$ & 0,13 \\
\hline
\end{tabular}

*missing: 9 na faixa etária entre $\geq 18$ e $<60,6$ entre $\geq 60$ e $<80$ e 3 nos $\geq 80$ anos ** teste Qui-Quadrado

$\mathrm{Na}$ Tabela 3, observa-se que os grupos diferiram na alta em relação às seguintes intervenções: Mobilização $e$ posicionamento, Suporte respiratório e Hiperalimentação intravenosa. 
Tabela 3 - Distribuição dos pacientes internados nas UTIs $(n=582 *)$ de acordo com a faixa etária e intervenção realizada na alta da unidade, conforme o NAS - São Paulo - 2006/2007

\begin{tabular}{|c|c|c|c|c|c|c|c|c|}
\hline \multirow{3}{*}{ Tipo de intervenções - NAS } & \multicolumn{7}{|c|}{ Faixas etárias } & \multirow{3}{*}{$p^{* *}$} \\
\hline & \multirow[t]{2}{*}{ Categorias } & \multicolumn{2}{|c|}{$\begin{array}{c}\text { Adultos } \\
(\geq 18 \mathrm{e}<60)\end{array}$} & \multicolumn{2}{|c|}{$\begin{array}{c}\text { Idosos } \\
(\geq 60 \mathrm{e}<\mathbf{8 0})\end{array}$} & \multicolumn{2}{|c|}{$\begin{array}{l}\text { Muito idosos } \\
\quad(\geq \mathbf{8 0})\end{array}$} & \\
\hline & & $\mathbf{N}$ & $\%$ & $\mathbf{N}$ & $\%$ & $\mathbf{N}$ & $\%$ & \\
\hline 1- Monitorização e controles & $\begin{array}{c}1 \mathrm{a} \\
1 \mathrm{~b} \text { e c }\end{array}$ & $\begin{array}{c}184 \\
86\end{array}$ & $\begin{array}{l}68,15 \\
31,85\end{array}$ & $\begin{array}{c}137 \\
73\end{array}$ & $\begin{array}{l}65,24 \\
34,76\end{array}$ & $\begin{array}{l}71 \\
31\end{array}$ & $\begin{array}{l}69,61 \\
30,39\end{array}$ & 0,69 \\
\hline 2- Investigações laboratoriais & $\begin{array}{l}\text { Sim } \\
\text { Não }\end{array}$ & $\begin{array}{c}254 \\
16\end{array}$ & $\begin{array}{c}94,07 \\
5,93\end{array}$ & $\begin{array}{c}203 \\
7\end{array}$ & $\begin{array}{c}96,67 \\
3,33\end{array}$ & $\begin{array}{c}100 \\
2\end{array}$ & $\begin{array}{c}98,04 \\
1,96\end{array}$ & 0,17 \\
\hline 3- Medicação & $\begin{array}{l}\text { Sim } \\
\text { Não }\end{array}$ & $\begin{array}{c}267 \\
3\end{array}$ & $\begin{array}{c}98,89 \\
1,11\end{array}$ & $\begin{array}{c}204 \\
6\end{array}$ & $\begin{array}{c}97,14 \\
2,86\end{array}$ & $\begin{array}{c}100 \\
2\end{array}$ & $\begin{array}{c}98,04 \\
1,96\end{array}$ & 0,38 \\
\hline 4- Procedimentos de higiene & $\begin{array}{c}4 \mathrm{a} \\
4 \mathrm{~b} \text { e c }\end{array}$ & $\begin{array}{c}229 \\
41\end{array}$ & $\begin{array}{l}84,81 \\
15,19\end{array}$ & $\begin{array}{c}175 \\
35\end{array}$ & $\begin{array}{l}83,33 \\
16,67\end{array}$ & $\begin{array}{l}83 \\
19\end{array}$ & $\begin{array}{l}81,37 \\
18,63\end{array}$ & 0,71 \\
\hline 5- Cuidados com drenos & $\begin{array}{l}\text { Sim } \\
\text { Não }\end{array}$ & $\begin{array}{l}149 \\
121\end{array}$ & $\begin{array}{l}55,19 \\
44,81\end{array}$ & $\begin{array}{c}122 \\
88\end{array}$ & $\begin{array}{l}58,10 \\
41,90\end{array}$ & $\begin{array}{l}61 \\
41\end{array}$ & $\begin{array}{l}59,80 \\
40,20\end{array}$ & 0,67 \\
\hline 6- Mobilização e posicionamento & $\begin{array}{c}6 \mathrm{a} \\
6 \mathrm{~b} \text { ec }\end{array}$ & $\begin{array}{c}171 \\
99\end{array}$ & $\begin{array}{l}63.33 \\
36,67\end{array}$ & $\begin{array}{c}99 \\
111\end{array}$ & $\begin{array}{l}47,14 \\
52,86\end{array}$ & $\begin{array}{l}55 \\
47\end{array}$ & $\begin{array}{l}53,92 \\
46,08\end{array}$ & $\mathbf{0 , 0 0}$ \\
\hline $\begin{array}{l}\text { 7- Suporte e cuidado aos familiares e } \\
\text { pacientes }\end{array}$ & $\begin{array}{l}7 \mathrm{a} \\
7 \mathrm{~b}\end{array}$ & $\begin{array}{c}266 \\
4\end{array}$ & $\begin{array}{c}98,52 \\
1,48\end{array}$ & $\begin{array}{c}202 \\
8\end{array}$ & $\begin{array}{c}96,19 \\
3,81\end{array}$ & $\begin{array}{c}99 \\
3\end{array}$ & $\begin{array}{c}97,06 \\
2,94\end{array}$ & 0,27 \\
\hline 8- Tarefas administrativas e gerenciais & $\begin{array}{c}8 \mathrm{a} \\
8 \mathrm{~b} \text { e c }\end{array}$ & $\begin{array}{c}243 \\
27\end{array}$ & $\begin{array}{l}90,00 \\
10,00\end{array}$ & $\begin{array}{c}176 \\
34\end{array}$ & $\begin{array}{l}83,81 \\
16,19\end{array}$ & $\begin{array}{c}93 \\
9\end{array}$ & $\begin{array}{c}91,18 \\
8,82\end{array}$ & 0,06 \\
\hline 9- Suporte respiratório & $\begin{array}{l}\text { Sim } \\
\text { Não }\end{array}$ & $\begin{array}{l}145 \\
125\end{array}$ & $\begin{array}{l}53,70 \\
46,30\end{array}$ & $\begin{array}{c}146 \\
64\end{array}$ & $\begin{array}{l}69,52 \\
30,48\end{array}$ & $\begin{array}{l}67 \\
35\end{array}$ & $\begin{array}{l}65,69 \\
34,31\end{array}$ & $\mathbf{0 , 0 0}$ \\
\hline 10- Cuidado com vias aéreas artificiais & $\begin{array}{l}\text { Sim } \\
\text { Não }\end{array}$ & $\begin{array}{c}50 \\
220\end{array}$ & $\begin{array}{l}18,52 \\
81,48\end{array}$ & $\begin{array}{c}52 \\
158\end{array}$ & $\begin{array}{l}24,76 \\
75,24\end{array}$ & $\begin{array}{l}28 \\
74\end{array}$ & $\begin{array}{l}27,45 \\
72,55\end{array}$ & 0,10 \\
\hline $\begin{array}{l}\text { 11- Tratamento para melhora da } \\
\text { função pulmonar }\end{array}$ & $\begin{array}{l}\text { Sim } \\
\text { Não }\end{array}$ & $\begin{array}{c}86 \\
184\end{array}$ & $\begin{array}{l}31,85 \\
68,15\end{array}$ & $\begin{array}{c}72 \\
138\end{array}$ & $\begin{array}{l}34,29 \\
65,71\end{array}$ & $\begin{array}{l}43 \\
59\end{array}$ & $\begin{array}{l}42,16 \\
57,84\end{array}$ & 0,17 \\
\hline 12- Medicação vasoativa & $\begin{array}{l}\text { Sim } \\
\text { Não }\end{array}$ & $\begin{array}{c}41 \\
229\end{array}$ & $\begin{array}{l}15,19 \\
84,81\end{array}$ & $\begin{array}{c}50 \\
160\end{array}$ & $\begin{array}{l}23,81 \\
76,19\end{array}$ & $\begin{array}{l}20 \\
82\end{array}$ & $\begin{array}{l}19,61 \\
80,39\end{array}$ & 0,06 \\
\hline $\begin{array}{l}\text { 13- Reposição intravenosa de grandes } \\
\text { perdas de fluidos }\end{array}$ & $\begin{array}{l}\text { Sim } \\
\text { Não }\end{array}$ & $\begin{array}{c}10 \\
260\end{array}$ & $\begin{array}{c}3,70 \\
96,30\end{array}$ & $\begin{array}{c}4 \\
206\end{array}$ & $\begin{array}{c}1,90 \\
98,10\end{array}$ & - & 100,00 & 0,10 \\
\hline 14- Monitorização de átrio esquerdo & $\begin{array}{l}\text { Sim } \\
\text { Não }\end{array}$ & $\begin{array}{c}5 \\
265\end{array}$ & $\begin{array}{c}1,85 \\
98,15\end{array}$ & $\begin{array}{c}5 \\
205\end{array}$ & $\begin{array}{c}2,38 \\
97,62\end{array}$ & - & 100,00 & 0,31 \\
\hline 15- Reanimação cardiorrespiratória & $\begin{array}{l}\text { Sim } \\
\text { Não }\end{array}$ & $\begin{array}{c}13 \\
257\end{array}$ & $\begin{array}{c}4,81 \\
95,19\end{array}$ & $\begin{array}{c}13 \\
197\end{array}$ & $\begin{array}{c}6,19 \\
93,81\end{array}$ & $\begin{array}{c}2 \\
100\end{array}$ & $\begin{array}{c}1,96 \\
98,04\end{array}$ & 0,26 \\
\hline 16- Técnicas de hemofiltração & $\begin{array}{l}\text { Sim } \\
\text { Não }\end{array}$ & $\begin{array}{c}19 \\
251\end{array}$ & $\begin{array}{c}7,04 \\
92,96\end{array}$ & $\begin{array}{c}11 \\
199\end{array}$ & $\begin{array}{c}5,24 \\
94,76\end{array}$ & $\begin{array}{c}6 \\
96\end{array}$ & $\begin{array}{c}5,88 \\
94,12\end{array}$ & 0,71 \\
\hline 17- Medida quantitativa do débito urinário & $\begin{array}{l}\text { Sim } \\
\text { Não }\end{array}$ & $\begin{array}{c}228 \\
42\end{array}$ & $\begin{array}{l}84,44 \\
15,56\end{array}$ & $\begin{array}{c}188 \\
22\end{array}$ & $\begin{array}{l}89,52 \\
10,48\end{array}$ & $\begin{array}{l}89 \\
13\end{array}$ & $\begin{array}{l}87,25 \\
12,75\end{array}$ & 0,26 \\
\hline 18- Medida de pressão intracraniana & $\begin{array}{l}\text { Sim } \\
\text { Não }\end{array}$ & $\begin{array}{c}4 \\
266\end{array}$ & $\begin{array}{c}1,48 \\
98,52\end{array}$ & $\begin{array}{c}2 \\
208\end{array}$ & $\begin{array}{c}0,95 \\
99,05\end{array}$ & - & 100,00 & 0,45 \\
\hline $\begin{array}{l}\text { 19- Tratamento de acidose/alcalose } \\
\text { metabólica complicada }\end{array}$ & $\begin{array}{l}\text { Sim } \\
\text { Não }\end{array}$ & $\begin{array}{c}4 \\
266\end{array}$ & $\begin{array}{c}1,48 \\
98,52\end{array}$ & $\begin{array}{c}3 \\
207\end{array}$ & $\begin{array}{c}1,43 \\
98,57\end{array}$ & $\begin{array}{c}1 \\
101\end{array}$ & $\begin{array}{c}0,98 \\
99,02\end{array}$ & 0,93 \\
\hline 20- Hiperalimentação intravenosa & $\begin{array}{l}\text { Sim } \\
\text { Não }\end{array}$ & $\begin{array}{c}13 \\
257\end{array}$ & $\begin{array}{c}4,81 \\
95,19\end{array}$ & $\begin{array}{c}30 \\
180\end{array}$ & $\begin{array}{l}14,29 \\
85,71\end{array}$ & $\begin{array}{c}7 \\
95\end{array}$ & $\begin{array}{c}6,86 \\
93,14\end{array}$ & $\mathbf{0 , 0 0}$ \\
\hline 21- Alimentação enteral & $\begin{array}{l}\text { Sim } \\
\text { Não }\end{array}$ & $\begin{array}{c}58 \\
212\end{array}$ & $\begin{array}{l}21,48 \\
78,52\end{array}$ & $\begin{array}{c}49 \\
161\end{array}$ & $\begin{array}{l}23,33 \\
76,67\end{array}$ & $\begin{array}{l}30 \\
72\end{array}$ & $\begin{array}{l}29,41 \\
70,59\end{array}$ & 0,27 \\
\hline 22- Intervenções específicas na unidade & $\begin{array}{l}\text { Sim } \\
\text { Não }\end{array}$ & $\begin{array}{c}4 \\
266\end{array}$ & $\begin{array}{c}1,48 \\
98,52\end{array}$ & $\begin{array}{c}3 \\
207\end{array}$ & $\begin{array}{c}1,43 \\
98,57\end{array}$ & $\begin{array}{c}1 \\
101\end{array}$ & $\begin{array}{c}0,98 \\
99,02\end{array}$ & 0,93 \\
\hline 23- Intervenções específicas fora da unidade & $\begin{array}{l}\text { Sim } \\
\text { Não }\end{array}$ & $\begin{array}{c}8 \\
262\end{array}$ & $\begin{array}{c}2,96 \\
97,04\end{array}$ & $\begin{array}{c}3 \\
207\end{array}$ & $\begin{array}{c}1,43 \\
98,57\end{array}$ & $\begin{array}{c}2 \\
100\end{array}$ & $\begin{array}{c}1,96 \\
98,04\end{array}$ & 0,52 \\
\hline
\end{tabular}

*missing: 9 na faixa etária entre $\geq 18$ e $<60,6$ entre $\geq 60$ e $<80$ e 3 nos $\geq 80$ anos

** teste Qui-Quadrado

Em relação à Mobilização e posicionamento, a comparação dos pares de grupos pelo Qui-Quadrado permitiu identificar que esta diferença ocorreu entre os grupos de adultos e idosos $(p=0,00)$, na qual os idosos apresenta- ram maior freqüência $(52,86 \%)$ dos itens $6 b$ e $6 c$ quando analisado perante os adultos $(36,67 \%)$, necessitando assim de maior tempo da enfermagem para a realização destes procedimentos. Não obstante, a diferença nos 
percentuais dos idosos $(52,86 \%)$ e muito idosos $(46,08 \%)$ e entre adultos $(36,67 \%)$ e muito idosos $(46,08 \%)$ não alcançou o nível de significância estabelecido neste estudo $(p \leq 0,05)$.

Quanto ao Suporte respiratório, o grupo de adultos diferiu dos idosos $(p=0,00)$ e dos muito idosos $(p=0,04)$. Os idosos e muito idosos necessitaram, em maior freqüência, do Suporte respiratório nas últimas 24 horas de internação na UTI, $69,52 \%$ e $65,69 \%$ respectivamente, do que os adultos $(53,70 \%)$.
A Hiperalimentação intravenosa foi mais freqüente nos idosos $(14,29 \%)$ e na comparação dos pares de grupos, observou-se que a diferença estatisticamente significativa ocorreu entre esse grupo e os adultos $(p=0,00)$ e esteve próxima ao nível de significância a comparação dos idosos e muito idosos $(p=0,06)$.

Cabe destacar que em apenas três dos 23 itens do NAS houve diferença estatisticamente significante entre os três grupos, na admissão e também na alta da UTI, conforme se observa nas Tabelas 2 e 3.

Tabela 4 - Diferença do NAS entre admissão e alta dos pacientes internados nas UTIs ( $n=600)$, dos sobreviventes ( $n=480)$ e dos que evoluíram a óbito na unidade (n=120), segundo faixa etária - São Paulo - 2006/2007

\begin{tabular}{|c|c|c|c|c|c|c|c|}
\hline \multirow{3}{*}{$\begin{array}{l}\text { NAS } \\
\text { Diferença admissão/alta }\end{array}$} & \multicolumn{6}{|c|}{ Faixas etárias } & \multirow{3}{*}{$\mathbf{p}^{*}$} \\
\hline & \multicolumn{2}{|c|}{$\begin{array}{c}\text { Adultos } \\
(\geq 18 \mathrm{e}<60)\end{array}$} & \multicolumn{2}{|c|}{$\begin{array}{c}\text { Idosos } \\
(\geq 60 \mathrm{e}<80)\end{array}$} & \multicolumn{2}{|c|}{$\begin{array}{c}\text { Muito idosos } \\
(\geq \mathbf{8 0})\end{array}$} & \\
\hline & Média & DP & Média & DP & Média & DP & \\
\hline Vivos e mortos & 9,61 & 20,16 & 8,56 & 22,74 & 9,05 & 21,26 & 0,86 \\
\hline Vivos & 11,27 & 19,54 & 12,71 & 21,48 & 10,91 & 21,83 & 0,74 \\
\hline Mortos & 0,77 & 21,36 & 3,59 & 22,13 & 1,59 & 17,35 & 0,49 \\
\hline
\end{tabular}

* Teste de Anova-OneWay

Na análise das diferenças médias do NAS entre admissão e alta, não houve diferença significante entre os três grupos quando foram analisados mortos e vivos conjuntamente e também em separado (Tabela 4).

\section{DISCUSSÃO}

Em relação às características da amostra total houve similaridade com outras investigações em relação as variáveis: idade ${ }^{(12-14)}$, sexo ${ }^{(12-17)}$, procedência(15-17), antecedentes $^{(15-17)}$, gravidade dos doentes ${ }^{(14,16)}$ e carga de trabalho de enfermagem na admissão na UTI(12,14-15,17). Valores de mortalidade e tempo de permanência em UTI, que apresentam grande variabilidade na literatura ${ }^{(12-17)}$, estiveram no intermédio, neste estudo, em relação aos descritos em outras pesquisas.

A idade da população tem aumentado progressivamente no Brasil ${ }^{(6)}$ assim com em outros países ${ }^{(5)}$ e a vivência clínica dos enfermeiros tem evidenciado a elevação do número de idosos em UTI em consonância com o aumento dessa população. Estudo realizado na Suíça confirmou esse pressuposto quando analisou, comparativamente, as características de 35.327 pacientes internados em UTIs entre os anos de 1980 e 1995 e mostrou que a idade dos pacientes aumentou significativamente no período ${ }^{(1)}$.

Embora já tenha sido descrito na literatura ${ }^{(7-8,18)}$ que a idade não determina um pior prognóstico, mas sim os fatores associados, tais como a gravidade da disfunção aguda e as co-morbidades, há ainda a percepção pelos profissionais da saúde que os idosos respondem menos a certas medidas terapêuticas e, portanto, requerem mais cuidados.
Não obstante, os resultados deste estudo revelam que o fator idade pouco interfere na carga de trabalho dispensada aos pacientes internados em UTIs corroborando com outras investigações que comparam pacientes idosos $(\geq 60$ anos) e não idosos ( $\geq 18$ e $<60$ anos) $)^{(15)}$ e somente de idosos de diferentes faixas etárias $(\geq 60$ e $<70 ; \geq 70$ e $<80$; $\geq 80)^{(16)}$. Além disso, pesquisas que analisaram a influência de diferentes variáveis (sexo, idade, tempo de permanência na UTI, mortalidade, gravidade, procedência e tipo de tratamento) nos valores do NAS evidenciaram que a idade não interferiu na demanda de carga de trabalho, reafirmando que investimentos terapêuticos são aplicados no sentido de promover a melhora clínica dos pacientes independente da faixa etária ${ }^{(12-13,17)}$.

A evolução da demanda de trabalho de enfermagem entre a admissão e alta da UTI (NAS admissão - NAS alta) e os valores do NAS na admissão foram similares entre os três grupos, podendo estar relacionados ao padrão de intervenções aplicado aos pacientes internados em UTI, independente da idade. No entanto, a carga de trabalho de enfermagem na alta mostrou-se diferente entre os grupos de adultos e idosos, indicando que, provavelmente, pacientes de idade mais avançada mantém demanda mais elevada de cuidados, após a alta da UTI.

Neste estudo foi interessante observar que as diferenças pontuais entre os três grupos recaíram nos procedimentos do NAS referentes às ações próprias de enfermagem (Monitorização e controles, Mobilização e posicionamento e Procedimentos de higiene) na admissão, enquanto que, na alta, essa diferença esteve presente nas intervenções que associam ações de outros profissionais (Suporte respiratório e Hiperalimentação intravenosa). Quanto a 
esses achados é importante salientar que o grupo de adultos foi aquele que exigiu menor carga de trabalho de enfermagem em relação a essas intervenções. Já os grupos de idosos e muito idosos alternaram na mais alta exigência de trabalho de enfermagem nesses itens.

Embora o número de itens do NAS que apresentaram diferença entre os grupos tenha sido pouco expressivo, os resultados demonstram que, principalmente em intervenções próprias de enfermagem, há uma maior demanda de trabalho por parte dos pacientes idosos e muito idosos que devem ser consideradas tanto no dimensionamento de pessoal da Unidade como na distribuição diária da equipe. Além disso, os pacientes com idade $\geq 60$ anos (idosos e muito idosos) exigiram e foram atendidos na sua maior demanda de cuidados, permitindo inferir que, uma vez admitidos na UTI, não há distinção em relação aos investimentos terapêuticos e de enfermagem.

\section{CONCLUSÃO}

Os resultados deste estudo apontaram que, independente da idade, houve similaridade da carga de traba-

\section{REFERÊNCIAS}

1. Jakob SM, Tothen HU. Intensive care 1980-1995: change in patient characteristics, nursing workload and outcome. Intensive Care Med. 1997;23(11):1165-70.

2. Miranda DR, Raoul N, Rijk A, Schaufeli W, lapichino G. Nursing Activities Score. Crit Care Med. 2003;31(2):374-82.

3. Telles SCR, Castilho V. Custo de pessoal na assistência direta de enfermagem em unidade de terapia intensiva. Rev Lat Am Enferm. 2007;15(5):1005-9.

4. Noseworthy TW, Konopad E, Shustack A, Johnston R, Grace $M$. Cost accouting of adult intensive care: methods and human and capital inputs. Crit Care Med. 1996;24(7):116872.

5. World Health Organization (WHO). WHO Study on Global AGEing and Adult Health (SAGE) [text on the Internet]. Geneva; 2009 [citado 2009 set.10]. Available from: www.who.int/healthinfo/systems/sage/en/index.html

6. Veras R. Population aging and health information from the National Household Sample Survey: contemporary demands and challenges. Introdução. Cad Saúde Pública. 2007;23(10):2463-6.

7. Galzerano A, Sabatini E, Durì D, Rossi C, Silvestri L, Mazzon $D$, et al. Old patients in intensive care unit (ICU): What decisions to make? Arch Gerontol Geriatr. 2009;49(2):294-7.
Iho de enfermagem na admissão, bem como na evolução das demandas de cuidados dos pacientes internados em UTIs. Da mesma forma, não foram observadas diferenças em 20 dos 23 itens do NAS na admissão e na alta da unidade.

Não obstante, as seguintes diferenças foram evidenciadas na comparação dos três grupos de pacientes:

- Adultos e idosos em relação à pontuação total do NAS na alta e nas seguintes intervenções: Monitorização e controles na admissão, Suporte respiratório e Hiperalimentação intravenosa na alta, além de Mobilização e posicionamento tanto na alta quanto na admissão;

- Adultos e muito idosos nas intervenções: Procedimentos de higiene e Mobilização e posicionamento na admissão, além do Suporte respiratório na alta;

- Idosos e muito idosos somente quanto aos Procedimentos de higiene na admissão.

8. López-Soto A, Sacanella E, Castejón MP, Nicolás JM. EI anciano en la Unidade de Cuidados Intensivos. Rev Esp Geriatr Gerontol. 2009;44 Supl 1:27-33.

9. Brasil. Ministério da Saúde. Portaria n. 466/MS/SVS, de 4 de junho de 1998. Dispõe sobre o regulamento técnico para o funcionamento dos Serviços de Tratamento Intensivo. Diário Oficial da União, Brasília, 5 jun.1998.

10. Le Gall JR, Lemeshow S, Saulnier F. A new Simplified Acute Physiology Score (SAPS II) based on a European/North American multicenter study. JAMA.1993;270(24):2957-63.

11. Le Gall JR, Klar J, Lemeshow S, Saulnier F, Alberti C, Artigas $A$, et al. The Logistic Organ Dysfunction system: a new way to assess organ dysfunction in the Intensive Care Unit. JAMA. 1996;276(10):802-10.

12. Padilha KG, Sousa RMC, Queijo AF, Mendes AM, Miranda DR. Nursing Activities Score in the Intensive Care Unit: analysis of the related factors. Intensive Crit Care Nurs. 2008;24(3):197-204.

13. Gonçalves LA, Padilha KG. Fatores associados à carga de trabalho de enfermagem em Unidade de Terapia Intensiva. Rev Esc Enferm USP. 2007;41(4):645-52.

14. Queijo AF. Estudo comparativo da carga de trabalho de enfermagem em Unidades de Terapia Intensiva geral e especializadas, segundo o Nursing Activities Score (NAS) [tese]. São Paulo: Escola de Enfermagem, Universidade de São Paulo; 2008. 
15. Ciampone JT, Gonçalves LA, Maia FOM, Padilha KG. Necessidades de cuidados de enfermagem e intervenções terapêuticas em Unidade de Terapia Intensiva: estudo comparativo entre pacientes idosos e não idosos. Acta Paul Enferm. 2006;19(1):28-35.

16. Sousa CR, Gonçalves LA, Toffoleto MC, Leão K, Padilha KG. Preditores da demanda de trabalho de enfermagem para idosos internados em Unidade de Terapia Intensiva. Rev Lat Am Enferm. 2008;16(2):218-23.
17. Gonçalves LA, Garcia PC, Toffoleto MC, Telles SCR, Padilha KG. Necessidades de cuidados de enfermagem em Terapia Intensiva: evolução diária dos pacientes segundo o Nursing Activities Score (NAS). Rev Bras Enferm. 2006;59(1):56-60.

18. Feijó CAR, Bezerra ISAM, Peixoto Junior AA, Meneses FA. Morbimortalidade do idoso internado na unidade de terapia intensiva de hospital universitário de Fortaleza. Rev Bras Ter Intensiva. 2006;18(3):263-7. 